\title{
An Effective Method to Accurately Calculate the Phase Space Factors for $\beta^{-} \beta^{-}$Decay
}

\author{
Andrei Neacsu and Mihai Horoi \\ Department of Physics, Central Michigan University, Mount Pleasant, MI 48859, USA \\ Correspondence should be addressed to Andrei Neacsu; neacsla@cmich.edu
}

Received 21 December 2015; Accepted 28 March 2016

Academic Editor: Aurelio Juste

Copyright (C) 2016 A. Neacsu and M. Horoi. This is an open access article distributed under the Creative Commons Attribution License, which permits unrestricted use, distribution, and reproduction in any medium, provided the original work is properly cited. The publication of this article was funded by SCOAP ${ }^{3}$.

\begin{abstract}
Accurate calculations of the electron phase space factors are necessary for reliable predictions of double-beta decay rates and for the analysis of the associated electron angular and energy distributions. We present an effective method to calculate these phase space factors that takes into account the distorted Coulomb field of the daughter nucleus, yet it allows one to easily calculate the phase space factors with good accuracy relative to the most exact methods available in the recent literature.
\end{abstract}

\section{Introduction}

Double-beta decay $(\beta \beta)$ processes are of considerable importance for the study of neutrinos. They change the charge $Z$ of a nucleus by two units, releasing two electrons, while the mass $A$ remains unchanged. The $\beta \beta$ decay with two associated electron antineutrinos in the final state conserves the lepton number and is permitted within the standard model (SM). This process, called two-neutrino double-beta decay $(2 \nu \beta \beta)$, has been experimentally observed for several isotopes with transitions to both ground states and excited states of the daughter nuclei [1]. Should the lepton number conservation be violated, then theories beyond the standard model (BSM) predict that the $\beta \beta$ decay transition could occur without antineutrinos in the final state, called neutrinoless double-beta $(0 \nu \beta \beta)$, and this implies that the neutrino is a Majorana fermion [2]. The $0 v \beta \beta$ transitions have not yet been confirmed experimentally, but there are many recent experimental and theoretical efforts dedicated to their discovery. Recent reviews on this matter are in [3-5]. There are several mechanisms that could contribute to the $0 \nu \beta \beta$ decay rate, of which the simplest and most studied one involves the exchange of light Majorana neutrinos in the presence of lefthanded weak interaction. Other, more complex, mechanisms include contributions from right-handed currents $[6,7]$ and mechanisms involving supersymmetry $[5,8]$.
The phase space factors (PSF) that enter the $\beta \beta$ lifetimes expressions were considered for a long time as being accurately calculated (see, e.g., $[9,10])$. Recent reevaluations of the PSF, using methods that take into account the proton distributions distorting the Coulomb field of the daughter nucleus [11-14], have shown considerable differences in some cases when compared to the previous results $[9,10]$. A very recent paper [14] presents four of the different methods commonly used to calculate the PSF and compares their results for the case of $0 \nu \beta \beta$ ground state (g.s.) transitions. Table 1 and Figure 2 of [14] show that the Coulomb distortion of the electron wavefunction by inclusion of the finite nuclear size and electron screening effects can produce differences of up to $100 \%$, compared to the point-charge formalism of [9] (see, e.g., the $0 \nu \beta \beta$ PSF $G_{08}$ of ${ }^{150} \mathrm{Nd}$ in [14]). However, taking into account the charge distributions in the daughter nuclei and solving numerically the Dirac equation with finite nuclear size are very slow and plagued by convergence issues. This makes these complex methods unattractive for the calculations of electron angular and energy distributions, such as those presented in $[15,16]$.

In this paper, we propose an effective method for treating the distortion of the Coulomb field in the daughter nucleus. This method uses the well known formalism of [9] but provides accurate results that are in good agreement with those of [11-14]. This method could be particularly useful when 
performing complex investigations involving PSF to test BSM physics due to different possible underlying mechanisms contributing to the $0 v \beta \beta$ process. These investigations often involve calculations of electron distributions $[15,16]$, where components of the PSF enter the equations, and it is not possible to only use the tabulated values of [11-14].

The paper is organized as follows. Section 2 shows the formalism for $0 \nu \beta \beta$ transitions to ground states and for $2 \nu \beta \beta$ transitions to ground and excited states. In Section 3, we present our effective method for the treatment of the distorted Coulomb field in the daughter nucleus. Section 4 is dedicated to the results, and Section 5 shows our conclusions. Appendices A and B summarize the point-charge formalism from $[9,10]$ that we adjusted to calculate the $0 \nu \beta \beta$ and $2 \nu \beta \beta$ PSF.

\section{Brief Formalism of the $\beta \beta$ Decay}

For the $0 \nu \beta \beta$ decay, one usually writes the inverse half-life as products of electron PSF, nuclear matrix element (NME) that depends on the nuclear structure of the parent and that of the daughter nuclei, and lepton number violation (LNV) parameters of the BSM mechanisms taken into account. Considering the existence of right-handed currents, one would find several additional contributions to the decay rate $[7,9]$. The most studied mechanism is that of the light left-handed neutrino exchange, but other mechanisms could be of importance [5]. One popular model that includes contributions of righthanded currents is the left-right symmetric model [17, 18]. This model assumes the existence of heavy particles that are not included in the standard model (SM). Within this framework, the $0 \nu \beta \beta$ half-life expression is given by

$$
\begin{aligned}
& {\left[T_{1 / 2}^{0 \nu}\right]^{-1}=G_{01}^{0 v} g_{A}^{4} \mid M^{0 v} \eta_{\nu}+M^{0 N}\left(\eta_{N_{R}}^{L}+\eta_{N_{R}}^{R}\right)+\eta_{\lambda} X_{\lambda}} \\
& \quad+\left.\eta_{\eta} X_{\eta}\right|^{2}
\end{aligned}
$$

where $g_{A}$ is the axial-vector coupling strength, $\eta_{\nu}, \eta_{N_{R}}^{L}$, $\eta_{N_{R}}^{R}, \eta_{\lambda}$, and $\eta_{\eta}$ are neutrino physics parameters defined in [19], $M^{0 v}$ and $M^{0 N}$ are the light and heavy neutrinoexchange nuclear matrix elements $[5,20]$, and $X_{\lambda}$ and $X_{\eta}$ represent combinations of NME and phase space factors. $G_{01}^{0 v}$ is a phase space factor [10] that can be calculated with relatively good precision in most cases [11, 12, 14]. Other possible contributions, such as those of $R$-parity violating SUSY particle exchange $[5,20]$, are neglected here. With some simplifying notations, the half-life expression [9] (here, we omit the contribution from the $\eta_{N_{R}}^{L}$ and $\eta_{N_{R}}^{R}$ terms, which share the same PSF as $\eta_{\nu}^{2}$ term, $G_{01}^{2 v}$, and have the same energy and angular distribution as the $\eta_{\nu}$ term) is written as

$$
\begin{gathered}
{\left[T_{1 / 2}^{0 \nu}\right]^{-1}=g_{A}^{4}\left[C_{1} \eta_{\nu}^{2}+\cos \phi_{1} C_{2} \eta_{\nu} \eta_{\lambda}+\cos \phi_{2} C_{3} \eta_{\nu} \eta_{\eta}\right.} \\
\left.+C_{4} \eta_{\lambda}^{2}+C_{5} \eta_{\eta}^{2}+\cos \left(\phi_{1}-\phi_{2}\right) C_{6} \eta_{\lambda} \eta_{\eta}\right]\left|M_{\mathrm{GT}}^{0 \nu}\right|^{2}
\end{gathered}
$$

where $\phi_{1}$ and $\phi_{2}$ are the relative CP-violating phases [19] and $M_{\mathrm{GT}}^{0 \nu}$ is the Gamow-Teller part of the light left-handed neutrino-exchange NME. Different processes give rise to several contributions: $C_{1}$ comes from the left-handed leptonic currents, $C_{4}$ from the right-handed leptonic and righthanded hadronic currents, and $C_{5}$ from the right-handed leptonic and left-handed hadronic currents. The interference between these terms is represented by the $C_{2}, C_{3}$, and $C_{6}$ contributions. Neglecting the very small tensor contributions in the mass mechanism, the $C_{1-6}$ components are defined as products of PSF and NME [9]:

$$
\begin{aligned}
C_{1} & =G_{01}^{0 v}\left(1-\chi_{F}\right)^{2}, \\
C_{2} & =\left[G_{04}^{0 v} \chi_{1+}+G_{03}^{0 v} \chi_{2-}\right]\left(1-\chi_{F}\right), \\
C_{3} & =\left[G_{03}^{0 v} \chi_{2+}-G_{04}^{0 v} \chi_{1-}-G_{05}^{0 v} \chi_{P}+G_{06}^{0 v} \chi_{R}\right]\left(1-\chi_{F}\right), \\
C_{4} & =\left[G_{02}^{0 v} \chi_{2-}^{2}+\frac{1}{9} G_{04}^{0 v} \chi_{1+}^{2}-\frac{2}{9} G_{03}^{0 v} \chi_{1+} \chi_{2-}\right], \\
C_{5} & =G_{02}^{0 v} \chi_{2+}^{2}+\frac{1}{9} G_{04}^{0 v} \chi_{1-}^{2}-\frac{2}{9} G_{03}^{0 v} \chi_{1-} \chi_{2+}+G_{08}^{0 v} \chi_{P}^{2} \\
& -G_{07}^{0 v} \chi_{P} \chi_{R}+G_{09}^{0 v} \chi_{R}^{2}, \\
C_{6} & =-2\left[G_{02}^{0 v} \chi_{2-} \chi_{2+}-\frac{1}{9} G_{03}^{0 v}\left(\chi_{1+} \chi_{2+}+\chi_{2-} \chi_{1-}\right)\right. \\
& \left.+\frac{1}{9} G_{04}^{0 v} \chi_{1+} \chi_{1-}\right],
\end{aligned}
$$

with

$$
\begin{aligned}
& \chi_{1 \pm}=\chi_{\mathrm{GT}_{q}} \pm 3 \chi_{F_{q}}-6 \chi_{T_{q}} \\
& \chi_{2 \pm}=\chi_{\mathrm{GT}_{\omega}} \pm \chi_{F_{\omega}}-\frac{\chi_{1 \pm}}{9}
\end{aligned}
$$

The fractions of NME are defined [9] as $\chi_{\alpha}=M_{\alpha} / M_{\mathrm{GT}}^{0 \nu}$, with $\alpha=F, \mathrm{GT}_{\omega}, F_{\omega}, \mathrm{GT}_{q}, F_{q}, T_{q}, R$, and $P$ indicating other NME. All these nine NME were calculated by several methods, including the interacting shell model (ISM) $[16,21$, 22] and quasiparticle random phase approximation (QRPA) [23]. The light-neutrino mass mechanism $M_{\mathrm{GT}}^{0 v}$ and $M_{F}^{0 v}$ NME have been extensively studied with many nuclear structure methods, such as interacting boson model (IBM2) [24-27], interacting shell model (ISM) [20, 21, 28-37], quasiparticle random phase approximation (QRPA) [3842], projected Hartree-Fock-Bogoliubov (PHFB) [43], energy density functional (EDF) [44], and the relativistic energy density functional (REDF) [45] method. The NME calculated with different methods and by different groups still present large differences, and that has been a topic of many debates in the literature (see, e.g., $[46,47])$. Expressions for the $G_{01}^{0 v}-G_{09}^{0 v}$ PSF are given in Appendix A.

For the $2 \nu \beta \beta$ process, the half-life for the transition to a state of angular momentum $J(J=0$ or 2$)$ of the daughter nucleus is given to a good approximation by [48]

$$
\left[T_{1 / 2}^{2 v}\right]^{-1}=G_{(J)}^{2 v} g_{A}^{4}\left|\left(m_{e} c^{2}\right)^{J+1} M_{(J)}^{2 v}\right|^{2},
$$


where $G_{(J)}^{2 v}$ is a phase space factor $[9,10,13]$ described in Appendix B, $m_{e}$ is the electron mass, and $M_{(J)}^{2 v}$ is the $2 v \beta \beta$ NME, which can be calculated as $[10,48]$

$$
M_{(J)}^{2 v}=\frac{1}{\sqrt{J+1}} \sum_{k} \frac{\left\langle J_{f}\left\|\sigma \tau^{-}\right\| 1_{k}^{+}\right\rangle\left\langle 1_{k}^{+}\left\|\sigma \tau^{-}\right\| 0_{i}^{+}\right\rangle}{\left(E_{k}+E_{J}\right)^{J+1}} .
$$

Here, the $k$-sum is taken over the $1_{k}^{+}$states with excitation energies $E_{k}$ in the intermediate odd-odd nucleus. Hence, $E_{J}=(1 / 2) Q_{\beta \beta}(J)+\Delta M$, where $Q_{\beta \beta}(J)$ is the $Q$-value for the transition to the state of angular momentum $J$ in the daughter nucleus, and $\Delta M$ is the difference in mass between the intermediate nucleus and the decaying nucleus.

\section{Description of the Effective "Screening" Method}

Our approach is based on the formalism from $[9,10]$ where the nuclear charge is considered point-like, but we replicate the effects of a finite size proton distribution distorting Coulomb field in the daughter nucleus by modifying the charge of the final nucleus $\left(Z_{f}\right)$. We multiply $Z_{f}$ with a parameter, called "screening factor" $\left(S_{f}\right)$ in what follows, to obtain an effective "screened charge" $\left(Z_{s}=\left(S_{f} / 100\right) Z_{f}\right)$. For large enough energies, the tail of the Coulomb field plays a less significant role when compared to its part close to the nucleus, and the effect resembles charge screening. The PSF calculated with $Z_{s}$ for each nucleus are compared to those of [11-14] (called "data" below), which were obtained with methods that consider Dirac electron wave functions calculated with finite nuclear size and atomic electron screening. References [11, 14] take into account the finite nuclear size by a uniform charge distribution of radius $R$, while $[12,13]$ consider a more realistic Woods-Saxon proton distribution inside the nucleus. It was shown [11] that the atomic electron screening effect is small, of the order of $0.1 \%$. The relative deviations between our results and the data, expressed in percentages $(\Delta=100 \mid(\mathrm{PSF}-$ data $) /$ datal $)$, are denoted by $\Delta G_{01-09}^{0 \nu}$ for the 9 PSF of $0 \nu \beta \beta$ transitions to ground states, $\Delta G_{\text {g.s. }}^{2 v}$ for PSF of $2 \nu \beta \beta$ transitions to ground states, $\Delta G_{0_{1}^{+}}^{2 v}$ for PSF of $2 \nu \beta \beta$ transitions to the first excited $0^{+}$state, and $\Delta G_{2_{1}^{+}}^{2 v}$ for PSF of $2 \nu \beta \beta$ transitions to the first excited $2^{+}$state.

The PSF can be grouped into two classes: those that have only $s$-wave electron contributions $\left(G_{01}^{0 \nu}, G_{\text {g.s. }}^{2 v}, G_{0_{1}^{+}}^{2 v}, G_{2_{1}^{+}}^{2 v}\right)$ and those to which $p$-wave electrons contribute $\left(G_{02}^{0 \nu}-G_{09}^{0 \nu}\right)$. Here, we treat them separately naming them $s$-PSF and $p$ PSF, respectively. We consider the largest deviation $\left(\Delta_{\max }\right)$ between the PSF of a certain class and the corresponding data, and we search for the value of $S_{f}$ that minimizes it. Our goal is to maintain $\Delta_{\max } \leq 10 \%$. This value of the maximum deviation is considerably lower than the uncertainties of the NME contributing to the decay rate, (2). We find that controlling the maximum deviation provides more stable and predictable results than minimizing a $\chi^{2}$ distribution.

In our analysis, data is selected as follows: $G_{01-09}^{0 \nu}$ PSF are chosen from Table III of [14]. Other recent results for $G_{01}^{0 \nu}$ [11-13] are within a few percent of these values. For $G_{02}^{0 v}-G_{09}^{0 \nu}$,

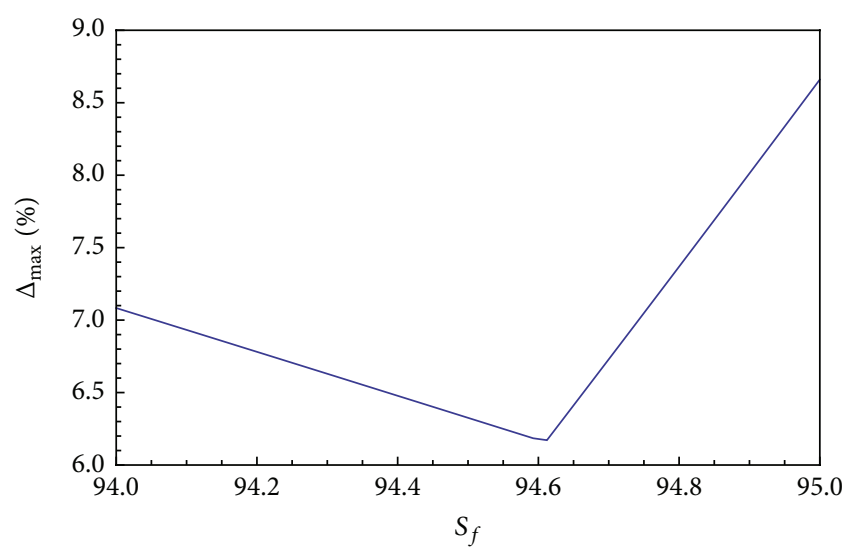

Figure 1: Fine-tuning the "screening factor" to minimize the maximum $s$-PSF deviations.

there are no other results that take into account the finite size effects of the charge distribution. The $G_{\text {g.s. }}^{2 v}$ data is taken from Table 1 of the very recent research [13], and it is in very good agreement with the results of [11]. For the $2 \nu \beta \beta$ transitions to the first excited $0^{+}$states, we take the data from Table 2 of [13]. There are four cases $\left({ }^{110} \mathrm{Pd},{ }^{124} \mathrm{Sn},{ }^{130} \mathrm{Te}\right.$, and $\left.{ }^{136} \mathrm{Xe}\right)$ of PSF in [13] that are in significant disagreement with those of [11]. We do not take them into account in our analysis. The data for $2 \nu \beta \beta$ transitions to the first excited $2^{+}$states is taken from Table 3 of [13]. In this case, there are three PSF values $\left({ }^{116} \mathrm{Cd},{ }^{124} \mathrm{Sn}\right.$, and $\left.{ }^{136} \mathrm{Xe}\right)$ that seem to deviate significantly from the model results. These $2 \nu \beta \beta$ PSF were not confirmed by other groups, and they were often readjusted [49]. We do not include them in the analysis, but we compare them with our prediction in Table 3 . The $2 \nu \beta \beta$ data of ${ }^{124} \mathrm{Sn}$ attributed to [13] in Tables 1-3 was provided to us as private communications by the authors of [13].

\section{Results and Discussions}

For the analysis of the $s$-PSF, we consider $G_{01}^{0 \nu}$ of [14] and $G_{\text {g.s. }}^{2 v}, G_{0_{1}^{+}}^{2 v}$, and $G_{2_{1}^{+}}^{2 v}$ of [13]. We find that the smallest maximum deviations from the data can be obtained using an optimal "screening factor" $S_{f}=94.5$. Figure 1 shows how the maximum deviation reaches a minimum when one gets close to the optimal "screening factor." Table 1 presents $s$ PSF and their deviations $\Delta G_{01}^{0 \nu}$ and $\Delta G_{\text {g.s. }}^{2 v}$ for transitions to ground states. The adjusted charge of the daughter nucleus is also presented, together with the $Q_{\beta \beta}$ values. We find very good agreement for these $s$-PSF and the data, with deviations smaller than $5 \%$. Should one consider the pointcharge formalism [9], the largest deviation goes up to $40 \%$ for the case of $G_{01}^{0 \nu}$ of ${ }^{150} \mathrm{Nd}$ (see, e.g., Table 1, columns $A$ and D, of [14]). Table 2 shows PSF and deviations from the data for $2 \nu \beta \beta$ transitions to the first excited $0^{+}$states. The largest $\Delta G_{0_{1}^{+}}^{2 \nu}=5.4 \%$ was for ${ }^{116} \mathrm{Cd}$. The point-charge formalism deviations exceed 38\%. The PSF marked with the "*" symbol correspond to the four nuclei not included in our analysis. Our results for these cases can be considered 
TABLE 1: $0 \nu \beta \beta$ to ground states PSF $G_{01}^{0 v}$ expressed in $\mathrm{yr}^{-1} \times 10^{15}$ and $2 \nu \beta \beta$ to ground states $G_{\text {g.s. }}^{2 v}$ expressed in $\mathrm{yr}^{-1} \times 10^{20}$. Results are calculated with the optimal "screening factor" for $s$-PSF, $S_{f}=94.5$, and compared to those of [14] for $0 v \beta \beta$ and [13] for $2 v \beta \beta$. Second and third columns display the effective screened charge, $Z_{s}$, of the daughter nucleus and the energy of the decay, $Q_{\beta \beta}$.

\begin{tabular}{|c|c|c|c|c|c|c|c|c|}
\hline & $Z_{s}$ & $Q_{\beta \beta}[\mathrm{MeV}]$ & $G_{01}^{0 \nu}$ & $G_{01}^{0 v}[14]$ & $\Delta G_{01}^{0 v}$ & $G_{\text {g.s. }}^{2 v}$ & $G_{\text {g.s. }}^{2 v}[13]$ & $\Delta G_{\text {g.s. }}^{2 v}$ \\
\hline${ }^{48} \mathrm{Ca}$ & 20.79 & 4.272 & 24.55 & 24.83 & 1.1 & 1480.46 & 1553.6 & 4.7 \\
\hline${ }^{76} \mathrm{Ge}$ & 32.13 & 2.039 & 2.28 & 2.37 & 3.8 & 4.51 & 4.65 & 2.9 \\
\hline${ }^{82} \mathrm{Se}$ & 34.02 & 2.995 & 9.96 & 10.18 & 2.1 & 150.31 & 157.3 & 4.4 \\
\hline${ }^{96} \mathrm{Zr}$ & 39.69 & 3.35 & 20.45 & 20.62 & 0.8 & 642.0 & 674.4 & 4.8 \\
\hline${ }^{100} \mathrm{Mo}$ & 41.58 & 3.034 & 15.74 & 15.95 & 1.3 & 310.6 & 323.1 & 3.9 \\
\hline${ }^{110} \mathrm{Pd}$ & 45.36 & 2.018 & 4.66 & 4.83 & 3.5 & 12.78 & 13.25 & 3.6 \\
\hline${ }^{116} \mathrm{Cd}$ & 47.25 & 2.814 & 16.57 & 16.73 & 1.0 & 258.78 & 268.8 & 3.7 \\
\hline${ }^{124} \mathrm{Sn}$ & 49.14 & 2.289 & 8.87 & 9.06 & 2.1 & 51.45 & 50.4 & 2.1 \\
\hline${ }^{130} \mathrm{Te}$ & 51.03 & 2.527 & 14.10 & 14.25 & 1.0 & 142.73 & 144.2 & 1.0 \\
\hline${ }^{136} \mathrm{Xe}$ & 52.92 & 2.458 & 14.49 & 14.62 & 0.9 & 133.73 & 133.2 & 0.4 \\
\hline${ }^{150} \mathrm{Nd}$ & 58.59 & 3.371 & 66.00 & 63.16 & 4.5 & 3467.53 & 3539.7 & 2.0 \\
\hline
\end{tabular}

TABLE 2: PSF and their deviations for $2 \nu \beta \beta$ to the first excited $0^{+}$states and $G_{0_{1}^{+}}^{2 v}$ expressed in $\mathrm{yr}^{-1} \times 10^{22}$. The last two columns present PSF of [11] and their deviations. The results marked with "*” and $(*)$ symbols (see text for details) correspond to the nuclei not included in the analysis.

\begin{tabular}{|c|c|c|c|c|c|c|c|}
\hline & $Z_{s}$ & $Q_{\beta \beta}[\mathrm{MeV}]$ & $G_{0_{1}^{+}}^{2 v}$ & $G_{0_{1}^{+}}^{2 v}[13]$ & $\Delta G_{0_{1}^{+}}^{2 v}[13]$ & $G_{0_{1}^{+}}^{2 v}[11]$ & $\Delta G_{0_{1}^{+}}^{2 v}[11]$ \\
\hline${ }^{48} \mathrm{Ca}$ & 20.79 & 1.275 & 3.43 & 3.52 & 2.6 & 3.63 & 5.5 \\
\hline${ }^{76} \mathrm{Ge}$ & 32.13 & 0.917 & 0.64 & 0.61 & 5.1 & 0.70 & 7.7 \\
\hline${ }^{82} \mathrm{Se}$ & 34.02 & 1.508 & 41.94 & 41.7 & 0.6 & - & - \\
\hline${ }^{96} \mathrm{Zr}$ & 39.69 & 2.202 & 1633.8 & 1694 & 3.6 & 1754 & 6.9 \\
\hline${ }^{100} \mathrm{Mo}$ & 41.58 & 1.904 & 562.08 & 570.8 & 1.5 & 605.5 & 7.2 \\
\hline${ }^{110} \mathrm{Pd}^{*}$ & 45.36 & 0.547 & 0.043 & 0.033 & 30.9 & 0.048 & 10.8 \\
\hline${ }^{116} \mathrm{Cd}$ & 47.25 & 1.057 & 8.00 & 7.59 & 5.4 & 8.73 & 8.3 \\
\hline${ }^{124} \mathrm{Sn}^{(*)}$ & 49.14 & 1.120 & 15.09 & 14.1 & 7.0 & - & - \\
\hline${ }^{124} \mathrm{Sn}^{*}$ & 49.14 & 0.630 & 0.180 & - & - & 0.199 & 9.7 \\
\hline${ }^{130} \mathrm{Te}^{*}$ & 51.03 & 0.734 & 0.69 & 0.55 & 25.9 & 0.76 & 9.2 \\
\hline${ }^{136} \mathrm{Xe}^{*}$ & 52.92 & 0.979 & 3.31 & 2.82 & 17.2 & 3.62 & 8.7 \\
\hline${ }^{150} \mathrm{Nd}$ & 58.59 & 2.631 & 40637.5 & 41160 & 1.3 & 43290 & 6.1 \\
\hline
\end{tabular}

TABLE 3: PSF and their deviations for $2 \nu \beta \beta$ to the first excited $2^{+}$ states and $G_{2_{1}^{+}}^{2 v}$ expressed in $\mathrm{yr}^{-1} \times 10^{21}$. Denoted with “*” symbol are PSF and deviations corresponding to the nuclei not included in the analysis.

\begin{tabular}{lccccc}
\hline & $Z_{s}$ & $Q_{\beta \beta}[\mathrm{MeV}]$ & $G_{2_{1}^{+}}^{2 v}$ & $G_{2_{\perp}^{+}}^{2 v}[13]$ & $\Delta G_{2_{\perp}^{+}}^{2 v}$ \\
\hline${ }^{48} \mathrm{Ca}$ & 20.79 & 4.272 & 3816 & 4074 & 6.3 \\
${ }^{76} \mathrm{Ge}$ & 32.13 & 2.039 & 0.40 & 0.38 & 3.5 \\
${ }^{82} \mathrm{Se}$ & 34.02 & 2.995 & 71.16 & 69.6 & 2.2 \\
${ }^{96} \mathrm{Zr}$ & 39.69 & 3.35 & 730.8 & 742.5 & 2.0 \\
${ }^{100} \mathrm{Mo}$ & 41.58 & 3.034 & 585 & 569 & 2.8 \\
${ }^{110} \mathrm{Pd}$ & 45.36 & 2.018 & 0.46 & 0.46 & 0.8 \\
${ }^{116} \mathrm{Cd}^{*}$ & 47.25 & 1.520 & 2.11 & 1.88 & 12.4 \\
${ }^{124} \mathrm{Sn}^{*}$ & 49.14 & 1.686 & 8.89 & 7.63 & 16.5 \\
${ }^{130} \mathrm{Te}^{136} \mathrm{Xe}^{*}$ & 51.03 & 2.527 & 81.09 & 79.6 & 1.9 \\
${ }^{150} \mathrm{Nd}$ & 52.92 & 1.640 & 9.03 & 7.68 & 17.6 \\
\hline
\end{tabular}

as predictions for these cases that are not yet validated by other methods. The last two columns show the results of [11] and the corresponding deviations, for comparison. Reference [11] provides no value for ${ }^{82} \mathrm{Se}$. The case of ${ }^{124} \mathrm{Sn}$ is more complicated because of the different values used in the literature for the energy of the first excited $0^{+}$state (see [50] for details). We include here with $*$ the phase space factor corresponding to $Q_{\beta \beta}$ used in [11] and with (*) the phase space factor corresponding to $Q_{\beta \beta}$ considered in [51] (see discussion in [50]). The $G_{2_{1}^{+}}^{2 v}$ PSF and their deviations are displayed in Table 3. We find the largest deviation $\Delta G_{2_{1}^{+}}^{2 v}=$ $6.3 \%$ for ${ }^{48} \mathrm{Ca}$. Neglecting finite nuclear size effects, one would get a deviation of $47 \%$ for ${ }^{150} \mathrm{Nd}$. Similar to the previous table, the three results excluded from the analysis are presented for comparison and marked with the "*” symbol.

When calculating the $p$-PSF, $G_{02}^{0 v}-G_{09}^{0 v}$, we find a different optimal "screening factor", $S_{f}=92$, corresponding to a larger 
TABLE 4: The calculated $0 \nu \beta \beta$ PSF $\left(G_{01}^{0 \nu}-G_{09}^{0 \gamma}\right)$ expressed in $\mathrm{yr}^{-1}$ for the decay to ground state of the 11 nuclei listed in Table 1 . The last line shows the optimal "screening factor" $S_{f}$ for all 8 -PSF $\left(G_{02}^{0 \nu}-G_{09}^{0 \nu}\right)$. For $G_{01}$, the $s$-PSF optimal "screening factor", $S_{f}=94.5$ of Table 1 , was used. Shown in the last column are the maximum deviations between our calculations with the indicated parameters and the results from [14].

\begin{tabular}{lccccccccccccc}
\hline & ${ }^{48} \mathrm{Ca}$ & ${ }^{76} \mathrm{Ge}$ & ${ }^{82} \mathrm{Se}$ & ${ }^{96} \mathrm{Zr}$ & ${ }^{100} \mathrm{Mo}$ & ${ }^{110} \mathrm{Pd}$ & ${ }^{116} \mathrm{Cd}$ & ${ }^{124} \mathrm{Sn}$ & ${ }^{130} \mathrm{Te}$ & ${ }^{136} \mathrm{Xe}$ & ${ }^{150} \mathrm{Nd}$ & $S_{f}[\%]$ & $\Delta_{\max }[\%]$ \\
\hline$Q_{\beta \beta}[\mathrm{MeV}]$ & 4.272 & 2.039 & 2.995 & 3.350 & 3.034 & 2.018 & 2.814 & 2.289 & 2.527 & 2.458 & 3.371 & \\
$G_{01}^{0 v} \cdot 10^{14}$ & 2.454 & 0.228 & 0.997 & 2.045 & 1.574 & 0.466 & 1.657 & 0.887 & 1.410 & 1.449 & 6.600 & 94.5 & 4.5 \\
$G_{02}^{0 v} \cdot 10^{14}$ & 16.11 & 0.376 & 3.468 & 8.928 & 5.733 & 0.782 & 5.309 & 1.919 & 3.719 & 3.639 & 30.65 & 95.0 & 5.0 \\
$G_{03}^{0 v} \cdot 10^{15}$ & 18.45 & 1.233 & 6.671 & 14.50 & 10.72 & 2.548 & 10.96 & 5.254 & 8.853 & 8.967 & 47.87 & 93.0 & 6.1 \\
$G_{04}^{0 v} \cdot 10^{15}$ & 5.283 & 0.453 & 2.099 & 4.382 & 3.345 & 0.937 & 3.511 & 1.829 & 2.960 & 3.035 & 14.45 & 95.5 & 4.2 \\
$G_{05}^{0 v} \cdot 10^{13}$ & 3.134 & 0.559 & 2.011 & 4.139 & 3.464 & 1.337 & 4.003 & 2.427 & 3.694 & 3.895 & 15.27 & 90.0 & 4.5 \\
$G_{06}^{0 v} \cdot 10^{14}$ & 3.869 & 0.496 & 1.655 & 2.951 & 2.388 & 87.46 & 2.482 & 1.472 & 2.157 & 2.209 & 7.813 & 92.0 & 6.6 \\
$G_{07}^{0 v} \cdot 10^{14}$ & 2.790 & 0.268 & 1.161 & 2.432 & 1.885 & 0.566 & 1.984 & 1.052 & 1.663 & 1.703 & 7.799 & 91.0 & 6.0 \\
$G_{08}^{0 v} \cdot 10^{14}$ & 1.212 & 0.154 & 0.732 & 1.776 & 1.417 & 0.443 & 1.654 & 0.891 & 1.468 & 1.548 & 7.946 & 89.5 & 9.3 \\
$G_{09}^{0 v} \cdot 10^{14}$ & 15.97 & 1.172 & 4.647 & 8.471 & 6.399 & 1.863 & 6.131 & 3.211 & 4.884 & 4.878 & 20.09 & 94.0 & 4.2 \\
\hline$p$-PSF & & \multicolumn{7}{c}{ Common $S_{f}$ parameter for $G_{02}^{0 v}-G_{09}^{0 v}$} & & & 92.0 \\
\hline
\end{tabular}

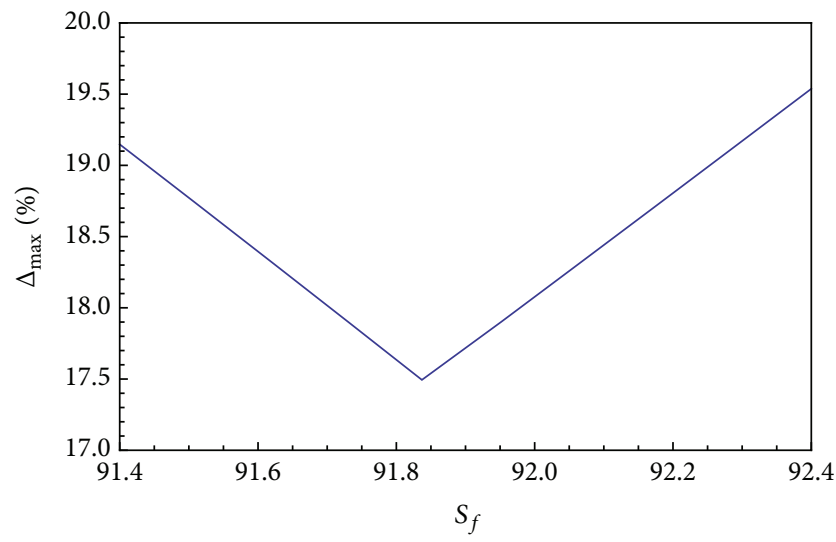

FIGURE 2: Same as Figure 1 for $p$-PSF.

maximum deviation, $\Delta_{\max }=18.1 \%$. Figure 2 presents the evolution of $\Delta_{\max }$ close to the "optimum screening factor" for $p$-PSF. We attribute this larger deviation to the different kinematic factors of the nine $0 \nu \beta \beta$ PSF (see (A.5a)-(A.5i)).

To further minimize the deviations, we obtain eight optimal "screening factors" corresponding to $G_{02}^{0 \nu}-G_{09}^{0 \nu}$, as seen in Figure 3. The best results are presented in Table 4, where we show the optimal "screening factor," the maximum deviations, and the PSF values. The last line presents the optimal "screening factor" that minimizes the deviations of all the $p$-PSF. Alongside $G_{02}^{0 v}-G_{09}^{0 v}$, we display $G_{01}^{0 v}$ obtained with the optimal "screening factor" common for all $s$-PSF.

\section{Conclusions}

In this paper, we present an effective method to calculate the phase space factors of the $\beta^{-} \beta^{-}$transitions, which can provide results close to those of methods that consider the finite size of the proton charge and the atomic electron screening. It modifies the point-charge formalism of $[9,10]$, by considering a constant multiplicative screening factor for the charge of the daughter nucleus. The main advantage of our method consists in its simplicity given its accuracy and its potential to be extended to calculations of the energy and angular electron distributions needed for the analysis of the contributions of the right-handed currents to the $0 \nu \beta \beta$ decay.

Our method works well for PSF of $0 \nu \beta \beta$ and $2 \nu \beta \beta$ transitions to ground states and also for $2 \nu \beta \beta$ transitions to the first excited $0^{+}$and $2^{+}$states. For PSF where only $s$-wave electrons contribute, an effective "screening factor", $S_{f}=$ 94.5, was obtained. Using this $S_{f}$ value, one finds a maximum deviation of $6.3 \%$ between our results and other results in the recent literature [11-14]. In the case of the PSF where $p$-wave electrons contribute, we obtained another optimal "screening factor", $S_{f}=92$. This corresponds to a maximum deviation of $18.1 \%$ between our results and those of [14]. We attribute this large deviation to the kinematic factors of (A.5a)-(A.5i). The deviations are greatly reduced, to less than $10 \%$, when considering individual "screening factors" for each specific PSF $\left(G_{02}^{0 \nu}-G_{09}^{0 \nu}\right)$. It is remarkable that, in the case of $G_{08}$, the original point-charge formalism [9] PSF deviates by over $100 \%$ for ${ }^{150} \mathrm{Nd}$, while it is significantly reduced in our model. Similar spectacular reductions are found for other $p$-PSF. We also provide predictions for the PSF of some isotopes, which can be also used as guidance in cases of disagreement between the more precise methods.

In addition, using $S_{f}=92$, one gets the largest maximum deviation of 18.1 for all neutrinoless double-beta decay PSF, $G_{01}^{0 \nu}-G_{09}^{0 \nu}$. This information is relevant for the calculation of the two-electron energy and angular distributions [16].

We conclude that this method is well suited for fast and accurate calculations of the $\beta \beta$ decay PSF, with uncertainties much lower than those of the associated NME. One could envision further reducing these PSF uncertainties by considering a mass-dependent screening factor. A Mathematica notebook that can be used to obtain all these phase space factors can be downloaded from [52]. 


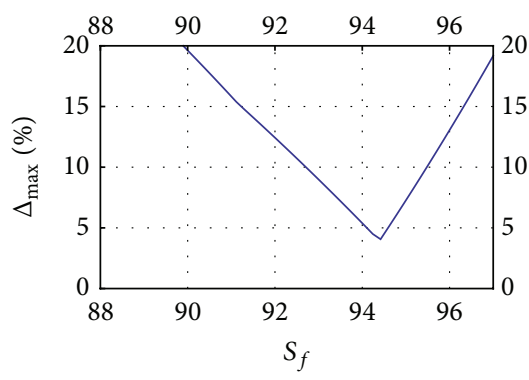

(a)

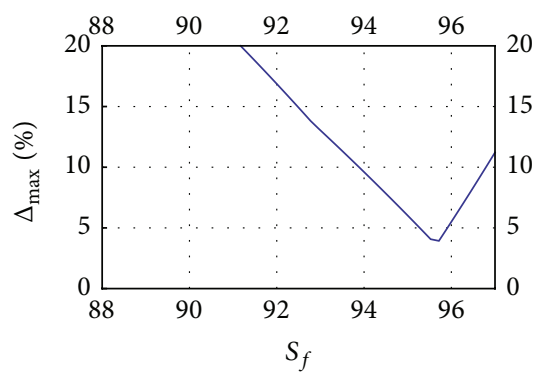

(d)

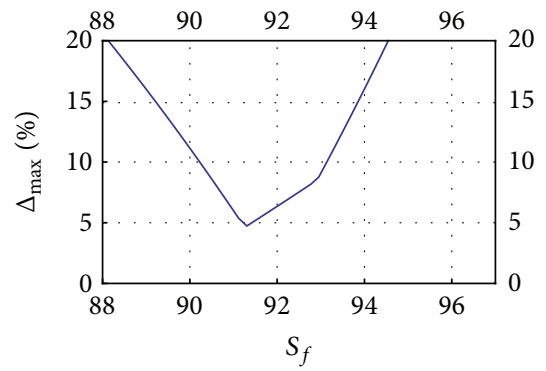

(g)

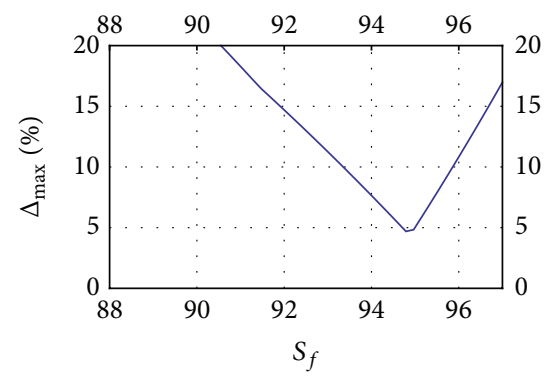

(b)

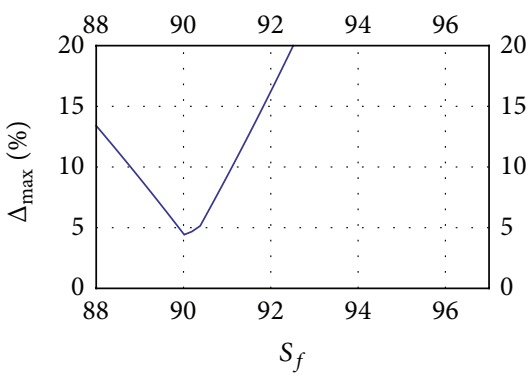

(e)

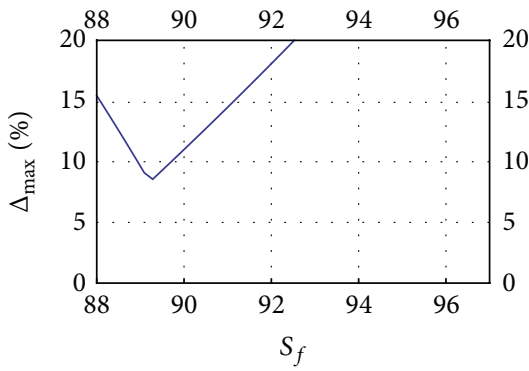

(h)

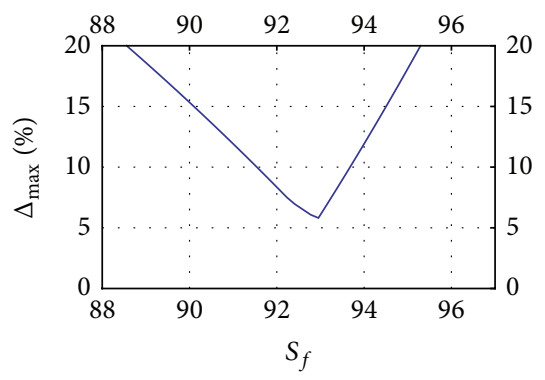

(c)

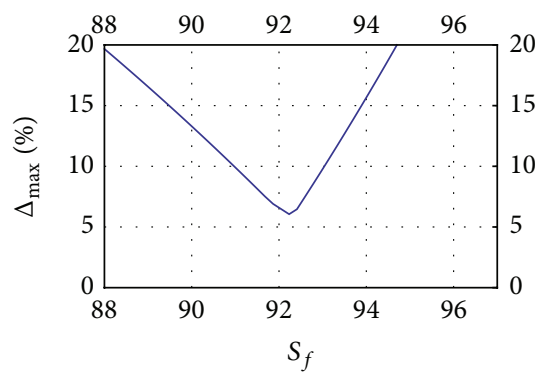

(f)

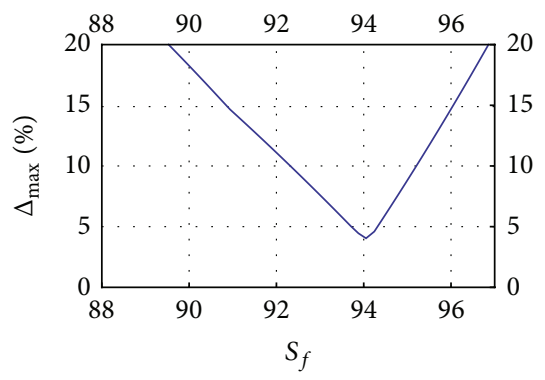

(i)

FIgURE 3: The behaviour of maximum deviations from the reference values of the 9 PSF involved in the $0 \nu \beta \beta$ transitions to ground states. Subfigures (a) to (i) correspond to $G_{01}^{0 \nu}$ to $G_{09}^{0 v}$. The horizontal axis represents the "screening factor" values, and the vertical axis represents the maximum deviations expressed in percentages.

\section{Appendix}

\section{A. $0 \nu \beta \beta$ PSF Expressions}

The $0 \nu \beta \beta$ PSF are calculated by integrating over the energy of one electron $\left(\epsilon_{1}\right)$ using the following expression adopted from equation (A.27) of [10]:

$$
\begin{aligned}
G_{0 k}= & \frac{a_{0 v}}{\ln 2\left(m_{e} R\right)^{2}} \\
& \cdot \int_{1}^{T+1} b_{k} F_{0}\left(Z, \epsilon_{1}\right) F_{0}\left(Z, \epsilon_{2}\right) \omega_{0 \nu}\left(\epsilon_{1}\right) d \epsilon_{1},
\end{aligned}
$$

where $R$ is the nuclear radius $\left(R=r_{0} A^{1 / 3}\right.$, with $\left.r_{0}=1.2 \mathrm{fm}\right)$, $\epsilon_{2}=T+2-\epsilon_{1}, p_{1,2}=\sqrt{\epsilon_{1,2}^{2}-1}, T=Q_{\beta \beta} / m_{e}$, and $\omega_{0 v}\left(\epsilon_{1}\right)=$ $p_{1} p_{2} \epsilon_{1} \epsilon_{2}$. The constant $a_{0 v}$ is

$$
a_{0 v}=\frac{\left(G_{F} \cos \theta_{c}\right)^{4} m_{e}^{9}}{32 \pi^{5}}=1.94 \times 10^{-22} \mathrm{yr}^{-1} \text {. }
$$

We use $G_{F}=1.1663787 \times 10^{-5} \mathrm{GeV}^{-2}$ for the Fermi constant and $\cos \theta_{c}=0.9749$ for the Cabibbo angle. The Fermi function used in (A.1) is given by

$$
\begin{aligned}
F_{0} & \left(Z_{s}, \epsilon\right) \\
& =\left[\frac{2}{\Gamma\left(2 \gamma_{1}+1\right)}\right]^{2}(2 p R)^{2\left(\gamma_{1}-1\right)}\left|\Gamma\left(\gamma_{1}+i y\right)\right|^{2} e^{\pi y},
\end{aligned}
$$

where

$$
\begin{aligned}
\gamma_{1} & =\sqrt{1-\left(\alpha Z_{s}\right)^{2}}, \\
y & =\frac{\alpha Z_{s} \epsilon}{p} .
\end{aligned}
$$


Here, $\alpha$ is fine structure constant, and $Z_{s}$ represents the "screened" charge of the final nucleus. The kinematic factors $b_{k}$ are defined as

$$
\begin{aligned}
& b_{1}=1 \text {, } \\
& b_{2}=\frac{1}{2}\left(\frac{\epsilon_{1} \epsilon_{2}-1}{\epsilon_{1} \epsilon_{2}}\right)\left(\epsilon_{1}-\epsilon_{2}\right)^{2}, \\
& b_{3}=\frac{\left(\epsilon_{1}-\epsilon_{2}\right)^{2}}{\epsilon_{1} \epsilon_{2}} \text {, } \\
& b_{4}=\frac{2}{9}\left(\frac{\epsilon_{1} \epsilon_{2}-1}{\epsilon_{1} \epsilon_{2}}\right) \text {, } \\
& b_{5}=\frac{4}{3}\left(\frac{(T+2) \xi}{2 r_{A} \epsilon_{1} \epsilon_{2}}-\frac{\epsilon_{1} \epsilon_{2}+1}{\epsilon_{1} \epsilon_{2}}\right) \text {, } \\
& b_{6}=\frac{4(T+2)}{r_{A} \epsilon_{1} \epsilon_{2}} \text {, } \\
& b_{7}=\frac{16}{3} \frac{1}{r_{A} \epsilon_{1} \epsilon_{2}}\left(\frac{\epsilon_{1} \epsilon_{2}+1}{2 r_{A}} \xi-T-2\right) \text {, } \\
& b_{8}=\frac{2}{9} \\
& \cdot \frac{1}{r_{A}^{2} \epsilon_{1} \epsilon_{2}}\left[\left(\epsilon_{1} \epsilon_{2}+1\right)\left(\xi^{2}+4 r_{A}^{2}\right)-4 r_{A} \xi(T+2)\right] \text {, } \\
& b_{9}=\frac{8}{r_{A}^{2}}\left(\frac{\epsilon_{1} \epsilon_{2}+1}{\epsilon_{1} \epsilon_{2}}\right)
\end{aligned}
$$

with $\xi=3 \alpha Z_{s}+r_{A}(T+2)$ and $r_{A}=m_{e} R$.

\section{B. $2 \nu \beta \beta$ PSF Expressions}

Using the formalism from [10], we write the $2 \nu \beta \beta$ PSF for a final state of angular momentum $J(J=0,2)$ as integrals over the energies of the two emitted electrons

$$
G_{(J)}^{2 v}=g_{J} \int_{1}^{T+1} F_{0}\left(Z_{s}, \epsilon_{1}\right) p_{1} \epsilon_{1} I_{J}\left(T, \epsilon_{1}\right) d \epsilon_{1}
$$

with $I_{J}$,

$$
\begin{aligned}
I_{J}\left(T, \epsilon_{1}\right)= & \int_{1}^{T+2-\epsilon_{1}} F_{0}\left(Z_{s}, \epsilon_{2}\right) p_{2} \epsilon_{2} \\
& \cdot f_{J}\left(T+2-\epsilon_{1}-\epsilon_{2}\right)^{2+J} d \epsilon_{2} .
\end{aligned}
$$

Here, $\epsilon_{2}=T+2-\epsilon_{1}, p_{1,2}=\sqrt{\epsilon_{1,2}^{2}-1}, T=Q_{\beta \beta} / m_{e}$, and $F_{0}$ is defined in (A.3). In the case of $J=0$, we have $f_{0}=1$ and $g_{0}=3.78 \times 10^{-25} \mathrm{yr}^{-1}$. For $J=2$, then $f_{2}=\left(\epsilon_{1}-\epsilon_{2}\right)^{2}$ and $g_{2}=g_{0} / 7$.

\section{Competing Interests}

The authors declare that they have no competing interests.

\section{Acknowledgments}

The authors acknowledge the useful discussions with S. Stoica and M. Mirea. Support from the NUCLEI SciDAC Collaboration under U.S. Department of Energy Grant no. DESC0008529 is acknowledged. Mihai Horoi also acknowledges U.S. NSF Grant no. PHY-1404442.

\section{References}

[1] A. Barabash, "Average and recommended half-life values for two-neutrino double beta decay," Nuclear Physics A, vol. 935, pp. 52-64, 2015.

[2] J. Schechter and J. W. F. Valle, "Neutrinoless double- $\beta$ decay in $\mathrm{SU}(2) \times \mathrm{U}(1)$ theories," Physical Review D, vol. 25, no. 11, pp. 2951-2954, 1982.

[3] F. T. Avignone III, S. R. Elliott, and J. Engel, "Double beta decay, Majorana neutrinos, and neutrino mass," Reviews of Modern Physics, vol. 80, no. 2, pp. 481-516, 2008.

[4] H. Ejiri, "Double beta decays and neutrino nuclear responses," Progress in Particle and Nuclear Physics, vol. 64, no. 2, pp. 249257, 2010.

[5] J. D. Vergados, H. Ejiri, and F. Šimkovic, "Theory of neutrinoless double-beta decay," Reports on Progress in Physics, vol. 75, no. 10, Article ID 106301, 2012.

[6] R. N. Mohapatra and J. C. Pati, "Left-right gauge symmetry and an 'isoconjugate' model of CP violation," Physical Review D, vol. 11, article 566, 1975.

[7] M. Doi, T. Kotani, H. Nishiura, and E. Takasugi, "Double beta decay," Progress of Theoretical Physics, vol. 69, no. 2, pp. 602-635, 1983.

[8] M. Hirsch, H. V. Klapdor-Kleingrothaus, and S. G. Kovalenko, "Supersymmetry and neutrinoless double $\beta$ decay," Physical Review D, vol. 53, no. 3, pp. 1329-1348, 1996.

[9] M. Doi, T. Kotani, and E. Takasugi, "Double beta decay and majorana neutrino," Progress of Theoretical Physics Supplement, vol. 83, pp. 1-175, 1985.

[10] J. Suhonen and O. Civitarese, "Weak-interaction and nuclearstructure aspects of nuclear double beta decay," Physics Report, vol. 300, no. 3-4, pp. 123-214, 1998.

[11] J. Kotila and F. Iachello, "Phase-space factors for double- $\beta$ decay," Physical Review C, vol. 85, no. 3, Article ID 034316, 2012.

[12] S. Stoica and M. Mirea, "New calculations for phase space factors involved in double- $\beta$ decay," Physical Review $C$, vol. 88, no. 3, Article ID 037303, 2013.

[13] M. Mirea, T. Pahomi, and S. Stoica, "Values of the phase space factors involved in double beta decay," Romanian Reports in Physics, vol. 67, no. 3, article 872, 2015.

[14] D. Stefanik, R. Dvornicky, F. Simkovic, and P. Vogel, "The light neutrino exchange mechanism of the $0 \nu \beta \beta$-decay with left-and right-handed leptonic and hadronic currents revisited," Physical Review C, vol. 92, Article ID 055502, 2015.

[15] R. Arnold, C. Augier, J. Baker et al., "Probing new physics models of neutrinoless double beta decay with SuperNEMO," The European Physical Journal C, vol. 70, no. 4, pp. 927-943, 2010.

[16] M. Horoi and A. Neacsu, "Analysis of mechanisms thatcould contribute to the neutrinoless double-beta decay," http://arxiv.org/abs/1511.00670.

[17] R. N. Mohapatra and J. C. Pati, “"Natural” left-right symmetry," Physical Review D, vol. 11, no. 9, pp. 2558-2561, 1975. 
[18] G. Senjanovic and R. N. Mohapatra, "Exact left-right symmetry and spontaneous violation of parity," Physical Review D, vol. 12, no. 5, pp. 1502-1505, 1975.

[19] J. Barry and W. Rodejohann, "Lepton number and flavour violation in $\mathrm{TeV}$-scale left-right symmetric theories with large left-right mixing," Journal of High Energy Physics, vol. 2013, no. 9, article 153, 2013.

[20] M. Horoi, "Shell model analysis of competing contributions to the double- $\beta$ decay of ${ }^{48} \mathrm{Ca}$," Physical Review $C$, vol. 87 , no. 1 , Article ID 014320, 2013.

[21] J. Retamosa, E. Caurier, and F. Nowacki, "Neutrinoless double beta decay of ${ }^{48} \mathrm{Ca}$," Physical Review C, vol. 51, no. 1, pp. 371-378, 1995.

[22] E. Caurier, F. Nowacki, A. Poves, and J. Retamosa, "Shell model studies of the double $\beta$ decays of ${ }^{76} \mathrm{Ge},{ }^{82} \mathrm{Se}$, and ${ }^{136} \mathrm{Xe}$," Physical Review Letters, vol. 77, no. 10, article 1954, 1954.

[23] K. Muto, E. Bender, and H. V. Klapdor, "Nuclear structure effects on the neutrinoless double beta decay," Zeitschrift für Physik A: Atomic Nuclei, vol. 334, no. 2, pp. 187-194, 1989.

[24] J. Barea and F. Iachello, "Neutrinoless double- $\beta$ decay in the microscopic interacting boson model," Physical Review $C$, vol. 79, no. 4, Article ID 044301, 16 pages, 2009.

[25] J. Barea, J. Kotila, and F. Iachello, "Limits on neutrino masses from neutrinoless double- $\beta$ decay," Physical Review Letters, vol. 109, no. 4, Article ID 042501, 4 pages, 2012.

[26] J. Barea, J. Kotila, and F. Iachello, "Nuclear matrix elements for double- $\beta$ decay," Physical Review C-Nuclear Physics, vol. 87, no. 1, Article ID 014315, 2013.

[27] J. Barea, J. Kotila, and F. Iachello, “ $0 \nu \beta \beta$ and $2 \nu \beta \beta$ nuclear matrix elements in the interacting boson model with isospin restoration," Physical Review C, vol. 91, no. 3, Article ID 034304 , 2015.

[28] E. Caurier, J. Menéndez, F. Nowacki, and A. Poves, "Influence of pairing on the nuclear matrix elements of the neutrinoless $\beta \beta$ decays," Physical Review Letters, vol. 100, no. 5, Article ID 052503, 2008.

[29] J. Menéndez, A. Poves, E. Caurier, and F. Nowacki, "Disassembling the nuclear matrix elements of the neutrinoless $\beta \beta$ decay," Nuclear Physics A, vol. 818, no. 3-4, pp. 139-151, 2009.

[30] E. Caurier, G. Martínez-Pinedo, F. Nowacki, A. Poves, and P. A. Zuker, "The shell model as a unified view of nuclear structure," Reviews of Modern Physics, vol. 77, no. 2, pp. 427-488, 2005.

[31] M. Horoi and S. Stoica, "Shell model analysis of the neutrinoless double- $\beta$ decay of ${ }^{48}$ Ca," Physical Review $C$, vol. 81, no. 2, Article ID 024321, 7 pages, 2010.

[32] R. A. Sen'kov and M. Horoi, "Neutrinoless double- $\beta$ decay of ${ }^{48} \mathrm{Ca}$ in the shell model: closure versus nonclosure approximation," Physical Review C, vol. 88, no. 6, Article ID 064312, 2013.

[33] M. Horoi and B. A. Brown, "Shell-model analysis of the ${ }^{136} \mathrm{Xe}$ double beta decay nuclear matrix elements," Physical Review Letters, vol. 110, no. 22, Article ID 222502, 5 pages, 2013.

[34] R. A. Sen'Kov, M. Horoi, and B. A. Brown, "Neutrinoless double- $\beta$ decay of Se 82 in the shell model: beyond the closure approximation," Physical Review C-Nuclear Physics, vol. 89, no. 5, Article ID 054304, 2014.

[35] A. Neacsu and S. Stoica, "Study of nuclear effects in the computation of the $0 \nu \beta \beta$ decay matrix elements," Journal of Physics G: Nuclear and Particle Physics, vol. 41, no. 1, Article ID 015201, 2014.

[36] R. A. Sen'kov and M. Horoi, "Accurate shell-model nuclear matrix elements for neutrinoless double- $\beta$ decay," Physical Review C, vol. 90, no. 5, Article ID 051301, 5 pages, 2014.
[37] A. Neacsu and M. Horoi, "Shell model studies of the ${ }^{130} \mathrm{Te}$ neutrinoless double- $\beta$ decay," Physical Review $C$, vol. 91, no. 2 , Article ID 024309, 2015.

[38] F. Šimkovic, G. Pantis, J. D. Vergados, and A. Faessler, "Additional nucleon current contributions to neutrinoless double $\beta$ decay," Physical Review C, vol. 60, Article ID 055502, 1999.

[39] J. Suhonen and O. Civitarese, "Effects of orbital occupancies and spin-orbit partners on $0 \nu \beta \beta$-decay rates," Nuclear Physics $A$, vol. 847, no. 3-4, pp. 207-232, 2010.

[40] A. Faessler, A. Meroni, S. T. Petcov, F. Simkovic, and J. Vergados, "Uncovering multiple $C P$-nonconserving mechanisms of $(\beta \beta)_{0 v}$ decay," Physical Review D, vol. 83, no. 11, Article ID 113003, 2011.

[41] M. T. Mustonen and J. Engel, "Large-scale calculations of the double- $\beta$ decay of ${ }^{76} \mathrm{Ge},{ }^{130} \mathrm{Te},{ }^{136} \mathrm{Xe}$, and ${ }^{150} \mathrm{Nd}$ in the deformed self-consistent Skyrme quasiparticle random-phase approximation," Physical Review C, vol. 87, no. 6, Article ID 064302, 2013.

[42] A. Faessler, M. González, S. Kovalenko, and F. Šimkovic, "Arbitrary mass Majorana neutrinos in neutrinoless double beta decay," Physical Review D, vol. 90, no. 9, Article ID 096010, 7 pages, 2014.

[43] P. K. Rath, R. Chandra, K. Chaturvedi, P. Lohani, P. K. Raina, and J. G. Hirsch, "Neutrinoless $\beta \beta$ decay transition matrix elements within mechanisms involving light Majorana neutrinos, classical Majorons, and sterile neutrinos," Physical Review C, vol. 88, no. 6, Article ID 064322, 2013.

[44] T. R. Rodríguez and G. Martínez-Pinedo, "Energy density functional study of nuclear matrix elements for neutrinoless $\beta \beta$ decay," Physical Review Letters, vol. 105, no. 25, Article ID 252503, 2010.

[45] L. S. Song, J. M. Yao, P. Ring, and J. Meng, "Relativistic description of nuclear matrix elements in neutrinoless double$\beta$ decay," Physical Review C, vol. 90, no. 5, Article ID 054309, 2014.

[46] A. Faessler, V. Rodin, and F. Šimkovic, "Nuclear matrix elements for neutrinoless double-beta decay and double-electron capture," Journal of Physics G: Nuclear and Particle Physics, vol. 39, no. 12, Article ID 124006, 2012.

[47] P. Vogel, "Nuclear structure and double beta decay," Journal of Physics G: Nuclear and Particle Physics, vol. 39, no. 12, Article ID 124002, 2012.

[48] M. Horoi, S. Stoica, and B. A. Brown, "Shell-model calculations of two-neutrino double- $\beta$ decay rates of ${ }^{48} \mathrm{Ca}$ with the GXPF1A interaction," Physical Review C, vol. 75, no. 3, Article ID 034303, 2007.

[49] M. Mirea, T. Pahomi, and S. Stoica, "Phase space factors fordouble $\beta$ decay: an up-date," http://arxiv.org/abs/1411.5506.

[50] M. Horoi and A. Neacsu, "Shell model predictions for ${ }^{124} \mathrm{Sn}$ double- $\beta$ decay," Physical Review $C$, vol. 93, no. 2, Article ID 024308, 2015.

[51] J. Dawson, R. Ramaswamy, C. Reeve, J. R. Wilson, and K. Zuber, "A search for various double beta decay modes of tin isotopes," Nuclear Physics A, vol. 799, no. 1-4, pp. 167-180, 2008.

[52] http://people.cst.cmich.edu/horoilm/bbcodes/simple_bbdecay_ psf.nb. 

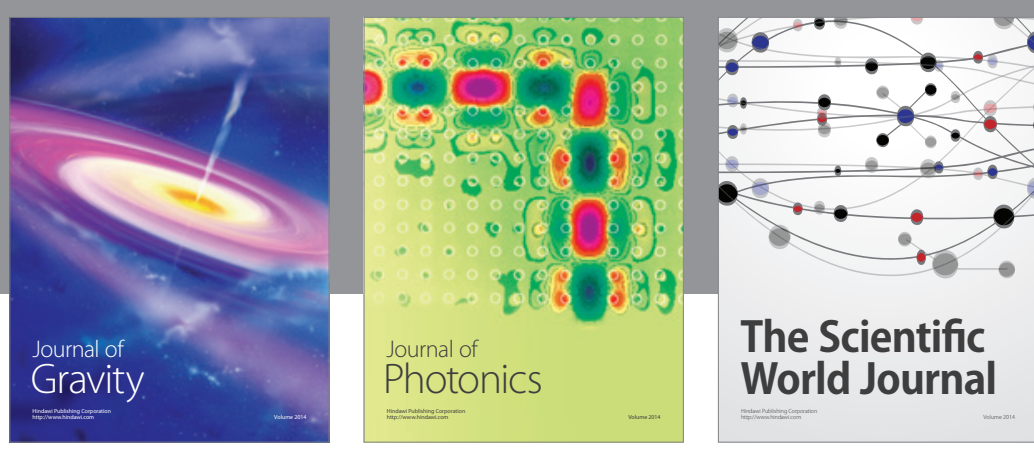

The Scientific World Journal
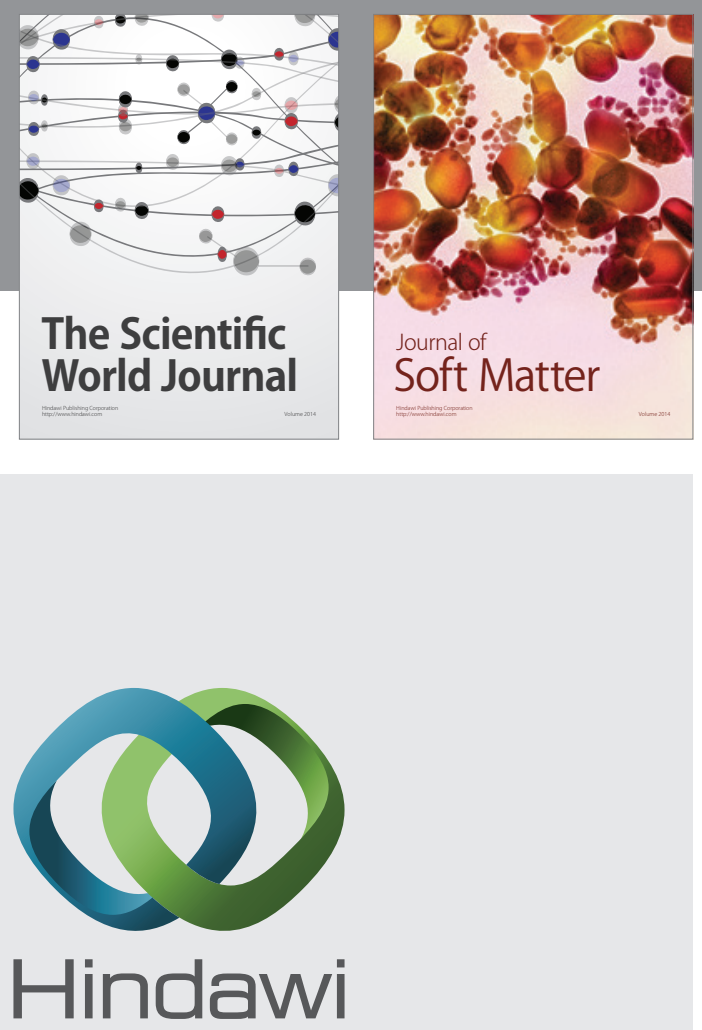

Submit your manuscripts at

http://www.hindawi.com

nternational Journal of

Statistical Mechanics
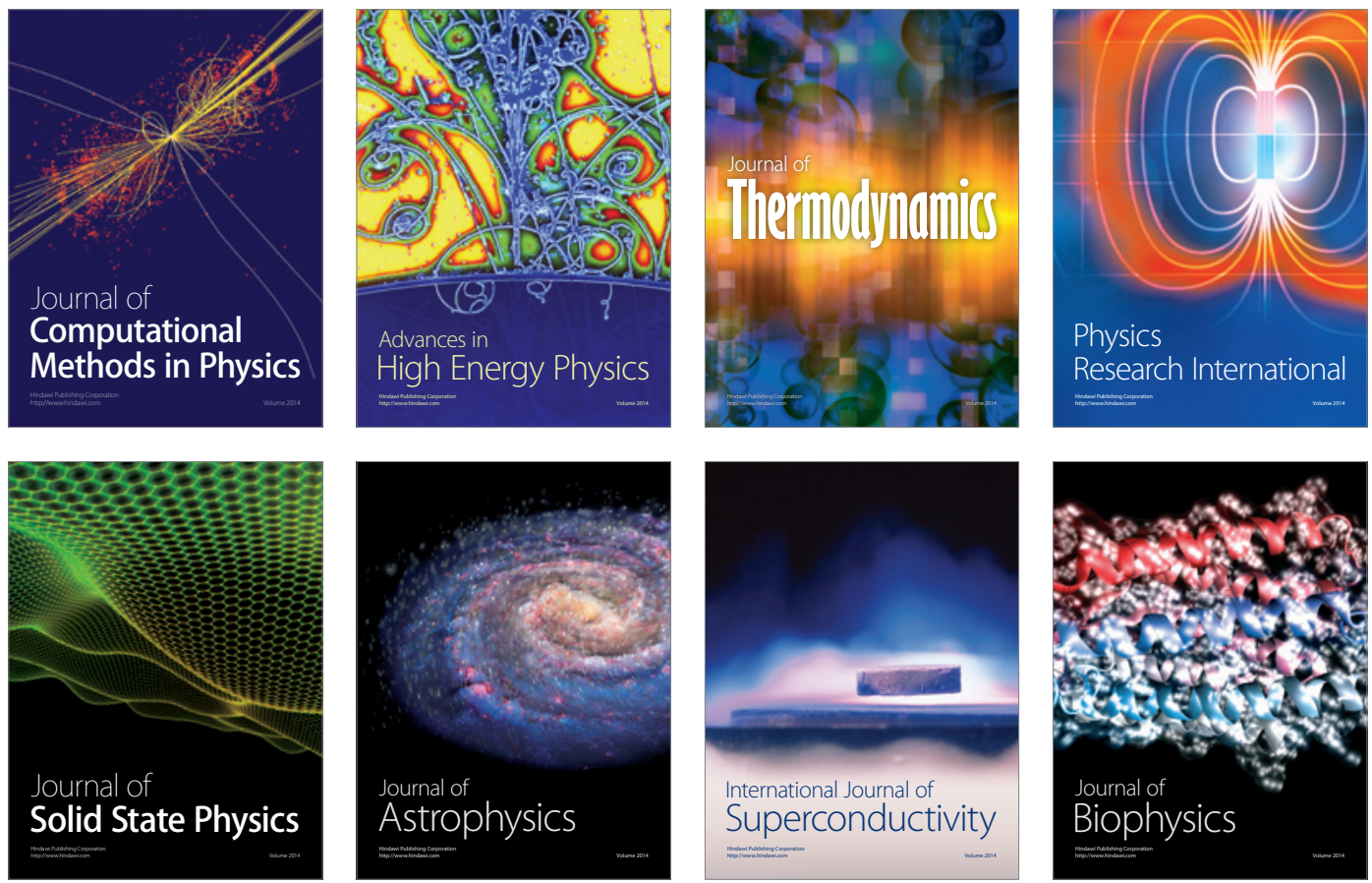
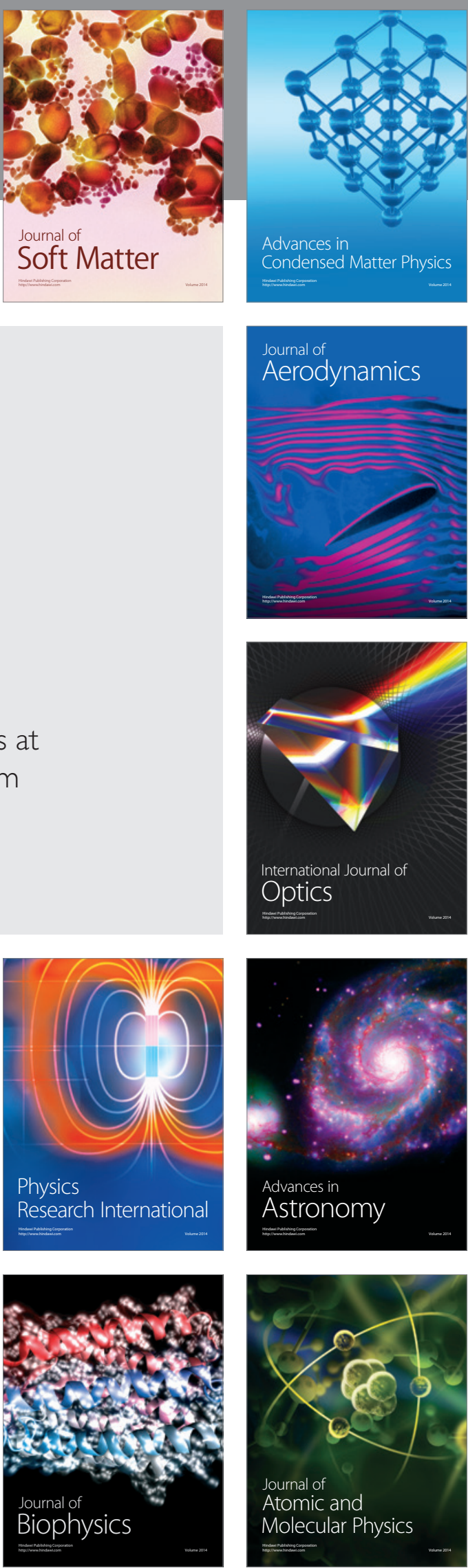